\title{
RELACION ENTRE DENSIDAD DE ESTOMAS Y LENTICELAS EN HOJAS Y FRUTOS DEL NOGAL (Juglans regia L.) Y LA SEVERIDAD DE Xanthomonas arboricola pv. juglandis
}

\section{RELATIONSHIP BETWEEN DENSITY OF STOMATA AND LENTICELS IN LEAVES AND FRUITS OF WALNUT (Juglans regia L.) AND THE SEVERITY OF Xanthomonas arboricola pv. juglandis}

\author{
Amelia Amanda Chorolque 1*,2, María Cristina Pozzo Ardizzi ${ }^{2}$ y Luis Francisco Hernández ${ }^{3,4}$ \\ ${ }^{1}$ Consejo Nacional de Investigaciones Científicas y Técnicas de Argentina (CONICET), Godoy Cruz \\ 2290, Buenos Aires (C1425FQB), Argentina. ORCID https://orcid.org/0000-0002-6363-8592 \\ ${ }^{2}$ Centro Universitario Regional Zona Atlántica, Universidad Nacional del Comahue, Monseñor Esandi \\ y Ayacucho s/n, Viedma (8500), Río Negro, Argentina. Correo electrónico: cpozzoar@gmail.com \\ ${ }^{3}$ Departamento de Agronomía, Universidad Nacional del Sur, San Andrés 800, Altos del Palihue, Bahía \\ Blanca (8000), Buenos Aires, Argentina. \\ ${ }^{4}$ Comisión de Investigaciones Científicas de la Provincia de Buenos Aires (CIC), La Plata (1900), Buenos \\ Aires, Argentina. Correo electrónico: lfhernandez@uns.edu.ar \\ *Autor para correspondencia E-mail: amelia.chorolque@curza.uncoma.edu.ar
}

\section{RESUMEN}

Xanthomonas arboricola pv. juglandis genera graves daños en diferentes cultivares de nogal. La bacteria se disemina por el agua y puede ingresar a través de entradas naturales de diferentes órganos de la planta. El objetivo de este trabajo fue analizar las características anatómicas de las hojas y los frutos de dos cultivares de nogal, Chandler y Franquette, y relacionarlas con la severidad de la enfermedad. La severidad de la enfermedad en la hoja (SevH) se determinó utilizando la escala de daño de Spielman, modificada. La densidad estomática (DE), el largo y el ancho de los estomas, se midieron utilizando un microscopio óptico, y el tamaño y la densidad de lenticelas (DL) en los frutos se midieron con un microscopio estereoscópico, ambos equipos con una escala ocular micrométrica. La severidad de la enfermedad en frutos (SevF) se evaluó mediante una escala que contempla 4 grados. Los resultados mostraron diferencias significativas en la SevH y en la DE entre los cultivares. El tamaño de los estomas presentó diferencias significativas solamente en la longitud, siendo mayor en cv. Chandler. Se determinó una fuerte correlación $(r=0,98)$ entre la SevH y la DE. Además, se observaron diferencias significativas entre cvs. Chandler y Franquette en términos de SevF y DL. No se observaron diferencias en el tamaño de las lenticelas. Los resultados permiten inferir que la DE y la DL son caracteres morfológicos a tener en cuenta cuando se analiza la susceptibilidad o tolerancia de cultivares de nogal frente a la peste negra del nogal.

Palabras clave: Juglans regia, estomas, lenticelas, nogal, tizón bacteriano, Xanthomonas.

\section{ABSTRACT}

Xanthomonas arborícola pv. juglandis generates severe damage to different walnut cultivars. The bacterium is carried through water and can enter plants through natural plant openings. The objective of this work was to analyze the anatomical characteristics of leaves and fruits of two walnut cultivars, Chandler and Franquette, and relate these traits to disease severity. Leaf disease severity (SevH) was determined using the modified Spielman's damage scale. Stomatal density (DE), length and width

Recibido: 03 octubre 2020. Aceptado: 06 noviembre 2020. 
of stomata were measured using a microscope, and the size and density of lenticels (DL) in the fruits were measured using a stereoscopic microscope, with an ocular micrometer. Fruit disease severity (SevF) was evaluated using a scale that included 4 levels of severity. The results showed significant differences in SevH and DE between cultivars. Stomatal size presented statistical differences only in length, being greater in Chandler. A strong correlation $(r=0.98)$ was found between SevH and DE. In addition, significant differences were observed between Chandler and Franquette in terms of SevF and DL. However, no differences were observed in lenticel size. The results obtained allow inferring that DE and DL should be taken into account when analyzing the susceptibility or tolerance of cultivars against walnut blight.

Key words: Bacterial blight, Juglans regia, lenticels, stomata, walnut, Xanthomonas

\section{INTRODUCCION}

El tizón bacteriano, o peste negra del nogal (Juglans regia L.), es una enfermedad causada por Xanthomonas arboricola pv. juglandis (Xaj); es la principal enfermedad que afecta a este frutal, causando severas pérdidas a nivel mundial. Ha sido reportada en la mayoría de las regiones productoras del mundo (Fernandes et al., 2017). En Argentina fue citada por primera vez por Marchionatto en 1944 (Flores et al., 2004; Temperini et al., 2014). En los valles irrigados de la Norpatagonia ha llegado a reducir en un $60-70 \%$ los rendimientos de los nogales (Nievas et al., 2014); en el Valle Inferior de Río Negro constituye la enfermedad más importante de este cultivo (Gallo, 2018).

Esta enfermedad produce daño en todos los órganos formados en la temporada de crecimiento. La bacteria inverna en las yemas durmientes (como epifítica o endofítica), en frutos enfermos que perduran en el árbol y en lesiones de ramas jóvenes (Flores et al., 2004; Lamichhane, 2014; Lindow et al., 2014; Nievas et al., 2014; Adaskaveg et al., 2016).

La mayoría de las bacterias ingresan a las diferentes partes de la planta a través de vías de entrada naturales, como estomas, nectarios y lenticelas de ramas jóvenes o el involucro de los frutos. La cantidad y disposición de estomas en las hojas depende de la especie de planta y de las condiciones ambientales en que se encuentre dicha hoja (Melotto et al., 2009; Naizaque et al., 2014). Para ingresar por las hojas, las bacterias necesitan una película de agua que facilite su introducción en la cavidad subestomática, donde se reproducen e inician la infección (Agrios, 2005).

Las lenticelas son estructuras localizadas en la epidermis de frutos, tallo, tubérculos, etc., con proliferación de células unidas en forma laxa, lo que permite el intercambio gaseoso en esos órganos. Durante la estación de crecimiento las lenticelas pueden permitir que las bacterias penetren en los tejidos, se desarrollen y avancen principalmente entre sus células (Agrios, 2005).
En primavera, cuando las condiciones ambientales favorecen la difusión de Xanthomonas arborícola pv. juglandis (Xaj), el riesgo de infección se incrementa en los diferentes cultivares de nogal. Estudios epidemiológicos han determinado que puede ingresar por estomas, lenticelas, estigmas y/o heridas (Mulrean y Schroth, 1982; Lopez et al., 2011). Se disemina a través del agua libre, por lo que lluvias, rocíos, nieblas, y/o riegos por aspersión, los que favorecen su dispersión. Si durante el período en que la hoja se mantiene mojada, la temperatura del aire estimula la tasa de crecimiento de Xaj, esto desencadena un período crítico para el hospedante que puede conducir a un proceso de infección (Belisario, 1996; Giovanardi et al., 2016; Temperini et al., 2017). Si bien existe una escala de susceptibilidad entre los cultivares crecidos en Argentina, todavía no se han desarrollado genotipos resistentes. Las variedades californianas de carga lateral (Vina, Chandler y Hartley) que son las más productivas, son también fuertemente afectadas, mientras que variedades europeas como Franquette o Mayette, de carga apical, producen menos frutos pero registran incidencias de la enfermedad significativamente menores (López et al., 2011; Nievas et al., 2014; Eskandari et al., 2018). Estas diferencias en el comportamiento frente a la enfermedad pueden deberse a diferencias en el desarrollo (las variedades californianas son de floración más temprana que las europeas), a la ubicación de los frutos sobre las ramas (laterales y apicales), o a diferencias anatómicas en las hojas $\mathrm{y}$ en los frutos (Aleta et al., 2001; Melotto et al., 2009; Bouhier, 2017).

Como la principal vía de ingreso de la bacteria son los estomas (en hojas) y las lenticelas en el involucro de los frutos (drupa involucrada) (Lang y Evans. 2010), es importante definir si los diferentes cultivares de nogal exhiben variabilidad en estos aspectos anatómicos, que puede condicionar su comportamiento frente a la enfermedad, y determinar la relación de la intensidad de la enfermedad con las características morfológicas y anatómicas de los 
órganos afectados por la bacteriosis.

El objetivo de este estudio fue determinar si la diferencia en la densidad y el tamaño de los estomas entre dos cultivares de nogal podrían constituir un indicador de susceptibilidad a Xanthomonas arboricola pv. juglandis, y paralelamente, analizar si las diferencias en la densidad y el tamaño de las lenticelas en el involucro del fruto (pericarpio), en cultivares susceptibles y tolerantes, se podrían relacionar con la severidad de la enfermedad.

\section{MATERIALES Y METODOS}

\section{Muestreo de cultivares de nogal}

Se estudiaron dos cultivares de nogal, Chandler (susceptible) y Franquette (tolerante), establecidos en un huerto en el valle inferior de Río Negro, Argentina (40 $47^{\prime}$ S.; $63^{\circ} 12^{\prime}$ O.). El diseño de plantación fue: $\mathrm{cv}$. Chandler, con un marco de plantación de $9 \times 7 \mathrm{~m}$ con polinizadoras del cv. Cisco, y cv. Franquette, con un marco de plantación de $7 \times 7 \mathrm{~m}$, con polinizadoras de la cV. IVARTO INTA. Estos cultivares fueron establecidos en el año 2005, conducidos en eje central modificado y con riego gravitacional por manto. La recolección de las muestras se realizó bajo un diseño experimental de bloques completamente aleatorizado con cuatro repeticiones.

\section{Determinación de la severidad de la enfermedad y características anatómicas sobre hojas y frutos}

La severidad de la enfermedad en hoja (SevH) se determinó sobre folíolos muestreados cada 45 días en la temporada 2016/17, desde noviembre hasta marzo. Se tomaron 4 muestras de 100 folíolos maduros recogidos al azar en la porción media de las hojas compuestas de las ramas jóvenes ubicadas entre 2 y 2,5 m del suelo de cada cultivar, y se fue evaluando el progreso de la enfermedad, clasificando los folíolos mediante la escala de severidad descripta por Spielman et al. (1986) que contempla 7 grados: 0: sana; 1 : entre 1 y $10 \%$ de superficie afectada (Saf); 2 : entre 11 y 25\% Saf; 3: 26-50\% Saf; 4: 51-75\% Saf; 5: 76-90\% Saf y 6: $91-100 \%$ Saf.

La SevH se calculó aplicando la siguiente fórmula:

$$
\operatorname{SevH}=\frac{\sum\left[\left(\mathrm{N}^{\circ} \text { de hojas } / \text { grado }\right) \times \text { grado }\right]}{\mathrm{N}^{\circ} \text { total de hojas } \times 7 \text { grados de severidad }} \times 100
$$

La Densidad Estomática (DE) y el tamaño de los estomas (ancho y largo) se midieron en folíolos muestreados en el mes de diciembre del 2017, cuando las hojas alcanzaron su máxima expansión. En 4 muestras de 50 folíolos cada una, se realizó una impresión epidérmica (Hernández y Green, 1993) en su cara abaxial para realizar el recuento estomático utilizando un microscopio óptico con ocular micrométrico (Zeiss, Modelo 4670589903, West Germany). En cada folíolo se contaron 3 campos tomados al azar. Por lo tanto el dato proveniente de cada muestra es el promedio de 150 registros.

La severidad de la enfermedad en fruto (SevF) se determinó cada 45 días sobre frutos muestreados en la temporada 2017/18, desde el mes de diciembre hasta el mes de marzo, en diferentes estadios fenológicos: primer muestreo cv. Chandler Gf (estigmas secos) y cv. Franquette Ff3 (inicio de oscurecimiento de estigmas); segundo muestreo, cáscara lignificada en el cv. Chandler y en el cv Franquette inicio de endurecimiento de la cáscara; tercer muestreo, cáscara totalmente lignificada del fruto en los dos cultivares, y cuarto muestreo en madurez de cosecha (proceso de apertura del pelón). En este último muestreo los frutos se recolectaron antes de la apertura total o dehiscencia del pelón (pericarpio) e inicio de la caída de la nuez al suelo. Los frutos de cada muestra se categorizaron según una escala preestablecida con 4 grados de severidad (Frutos et al., 2008; Fig. 1).

Para el cálculo de la SevF se utilizó la siguiente formula:

$\operatorname{SevF}=\frac{\sum\left[\left(\mathrm{N}^{\circ} \text { de frutos } / \text { grado }\right) \times \text { grado }\right]}{\mathrm{N}^{\circ} \text { total de frutos } \mathrm{x} 4 \text { grados de severidad }} \times 100$

La Densidad de Lenticelas (DL) y su tamaño se midieron en el receptáculo de frutos muestreados en diciembre de 2017, utilizando un microscopio estereoscópico con escala ocular (Arcano, Modelo ZTX 1:4, China). Se tomaron 20 frutos por cada tratamiento, en cada fruto se contaron 3 campos tomados al azar.

\section{Análisis estadístico}

Se realizó la comparación de los valores de la densidad estomática, la densidad de lenticelas y su tamaño mediante el Test de Student $(p<0,05)$. Además se construyeron las curvas de la $\mathrm{SevH}$ y la SevF en función del tiempo, linearizando su relación y se comparó la tasa de incremento de la enfermedad. Con los datos de SevH y los de DE, SevF y la DL, se realizó un análisis de regresión: SevH vs. DE y SevF vs. DL. Para el análisis estadístico se utilizó el software INFOSTAT (Di Rienzo, et al., 2008).

\section{RESULTADOS Y DISCUSIÓN}

\author{
Determinación de la severidad de la enfermedad \\ y características anatómicas de las hojas y los \\ frutos \\ La tasa de incremento de la enfermedad
}



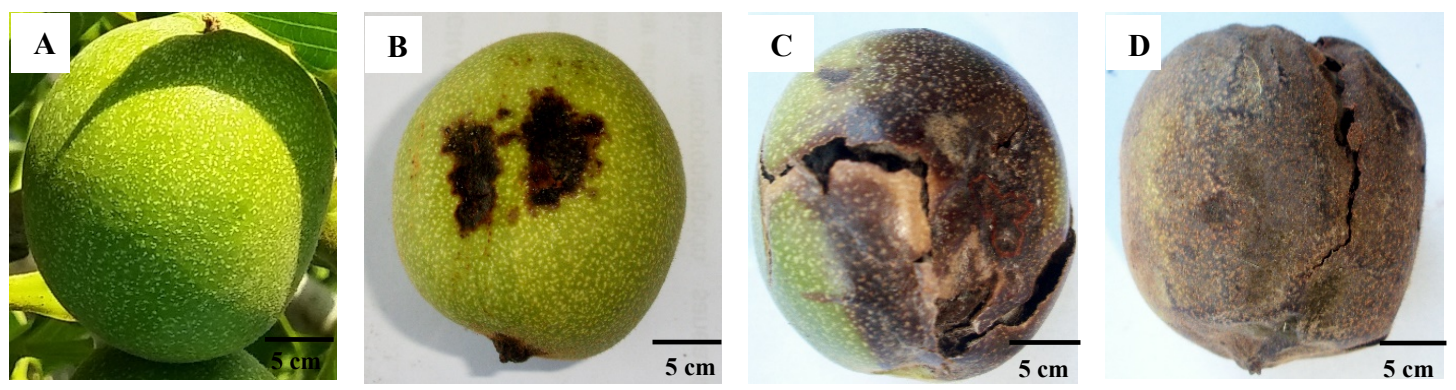

Fig. 1. Frutos de nogal en diferente estado de evolución de la enfermedad, mostrando los cuatro grados de severidad, según la escala descripta en Frutos et al. (2008). A. Grado 0: fruto sano; B. Grado 1: entre 1 y 10\% de superficie afectada; C. Grado 2: entre 11 y 40\% de superficie afectada; D. Grado 3: más del $40 \%$ de superficie afectada.

Fig. 1. Walnut fruits in different stages of disease evolution, showing the four severity levels according to the scale described in Frutos et al. (2008). A. Grade 0: healthy fruits; B. Grade 1: between 1 and $10 \%$ of the affected surface; C. Grade 2: between 11 and $10 \%$ of the affected surface; D Grade 3: more than $40 \%$ of the affected surface.

en hoja y fruto resultaron mayores en el cv. Chandler que en el cv. Franquette (Fig. 2 A y B), lo que determinó que, en el mes de marzo cuando finalizó la evaluación, la SevH y la SevF en 'Chandler', fueran estadísticamente superiores que en 'Franquette' (Tabla 1); siendo este último cultivar el de mejor comportamiento frente a la enfermedad y el 'Chandler' altamente susceptible. Estos resultados coinciden con lo observado por Aleta et al. (2002) y Flores et al. (2004).

Se observó una tendencia de la SevH a crecer exponencialmente (Fig. 2 A) mientras que la SevF se estabiliza en la madurez (Fig. 2 B). Estas diferentes dinámicas de evolución de la enfermedad se pueden explicar por las diferencias en el desarrollo de estos órganos. El fruto del nogal presenta un crecimiento del tipo sigmoide doble, donde en un momento se detiene su crecimiento total porque se está lignificando la cáscara de la nuez. Después de esto el fruto retoma la ganancia de peso, para finalmente dar comienzo a una etapa de senescencia. Se reconocen tres etapas: la primera llamada crecimiento rápido, una segunda, el endurecimiento del carozo o hueso, y una tercera y última etapa de maduración (Morales, 2012).

Se registraron diferencias significativas entre la densidad estomática, 'Chandler': 387 estomas $\mathrm{mm}^{-2}$;'Franquette': 315 estomas $\mathrm{mm}^{-2} \mathrm{y}$ su longitud (Tabla 1). Estas variaciones en las características estomáticas de los cultivares han sido reportado por diversos investigadores (Melotto et al., 2008; Lopez et al., 2011; Muradoğlu et al., 2011). En la Fig. 3 A y B se observa la distribución y el aspecto de los estomas en la epidermis abaxial en el cv. Chandler y el cv. Franquette.

El análisis sobre la SevF determinó que existen diferencias significativas entre cultivares. Esta variable fue mayor en 'Chandler' que en 'Franquette', 12,3\% y 6,2\%, respectivamente (Tabla 2). Estos resultados muestran que el cv. Franquette, de brotación tardía y de carga apical, es más tolerante frente a la enfermedad que el cv. Chandler, de brotación temprana y de carga lateral. Estos resultados coinciden con los reportado por Aleta et al. (2001), Flores et al. (2004), y Nievas et al. (2014).

La densidad de lenticelas en el fruto fue mayor en 'Chandler' $\left(131 \mathrm{~cm}^{-2}\right)$ y menor en 'Franquette' $\left(113 \mathrm{~cm}^{-2}\right)$, registrándose diferencias estadísticas. En el tamaño (longitud y ancho) de las lenticelas entre ambos cultivares no se observaron diferencias significativas (Tabla 2). En la Fig. 4 A y B se muestran los diferentes aspectos de las lenticelas en cada uno de los cultivares evaluados.

Análisis de regresión entre la severidad de la enfermedad en hoja vs. densidad estomática y la severidad de la enfermedad en fruto vs. densidad de lenticelas

Se determinó que existe una fuerte correlación entre la SevH y la DE de la cara abaxial (Fig. 5 A) dado que el Coeficiente de Correlación de Pearson fue $r=0,98$. La densidad estomática explica el 98 $\%$ de la SevH. Estos resultados estarían indicando que el número de estomas en la cara abaxial de la lámina más expuesta al inóculo primario (bacteria que sobrevive al invierno y que ocasiona las infecciones primarias), por su posición planófila, podría ser responsable de la mayor o menor incidencia de la enfermedad. Desde el punto de vista epidemiológico, la DE abaxial es la más importante cuando en los cultivares hay un predominio de hojas jóvenes (inicio de la 


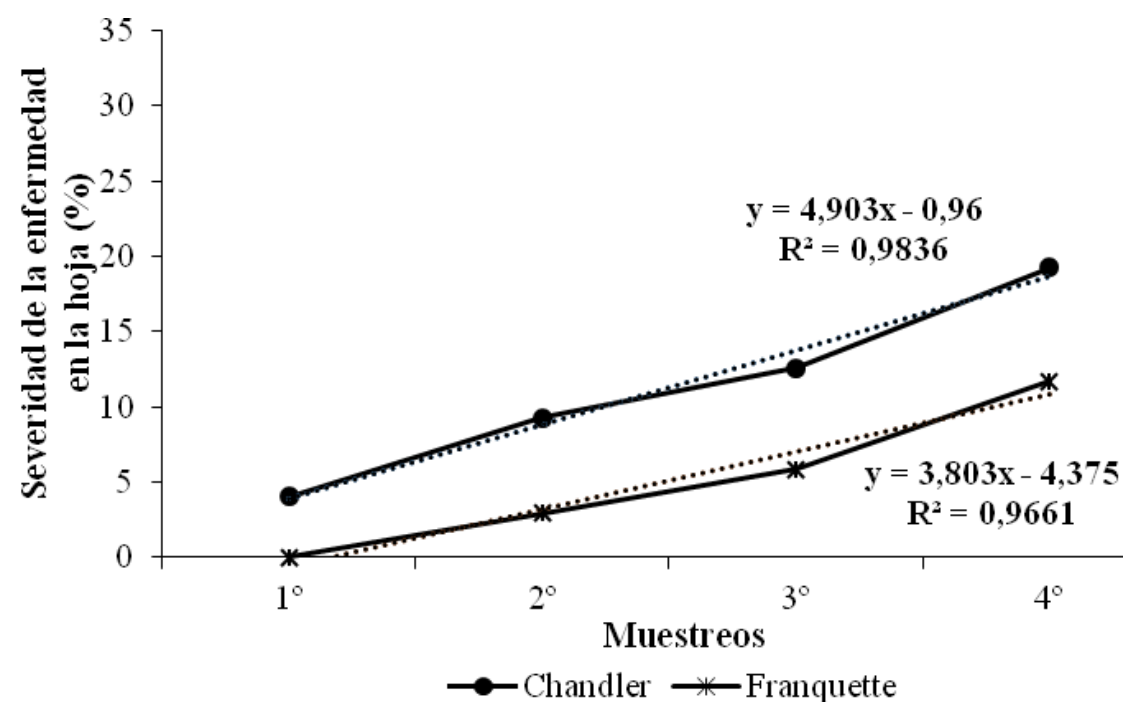

Fig. 2. A. Evolución de la severidad de la enfermedad en la hoja en los cultivares de nogal Chandler y Franquette durante la temporada 2016/2017.

Fig. 2. A. Evolution of leaf disease severity in the walnut cultivars Chandler and Franquette during the $2016 / 2017$ season.

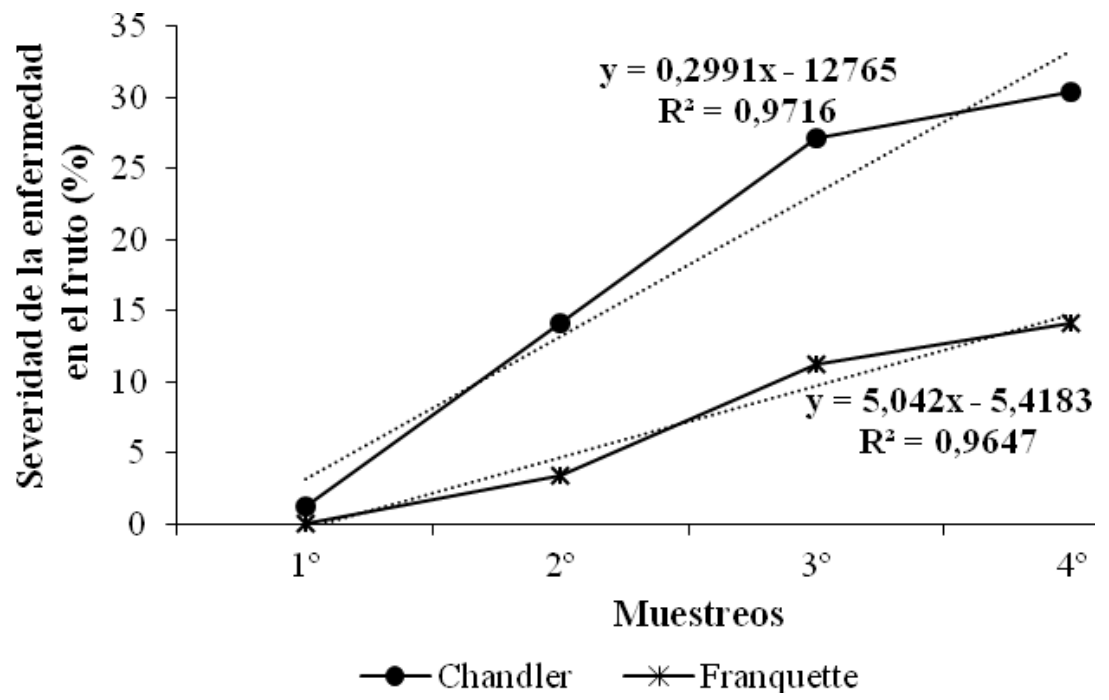

Fig. 2. B. Evolución de la severidad de enfermedad de frutos durante la temporada 2016/2017, en los cultivares de nogal Chandler y Franquette.

Fig. 2. B. Evolution of fruit disease severity in the walnut cultivars Chandler and Franquette during the 2016/2017 season.

temporada de crecimiento) pues, como el inóculo primario proviene de las escamas de las yemas dormidas y de cancros infectados de ramas del año anterior, el principal plano de impacto de la bacteria es la cara inferior de las hojas.
Los resultados mostraron un Coeficiente de Correlación de Pearson alto $(r=0,70)$ entre la SevF y la DL (Fig. 5. B), lo que significa que la diferencia en la DL explica el 70\% de la SevF. Si bien las lenticelas constituyen el principal punto 

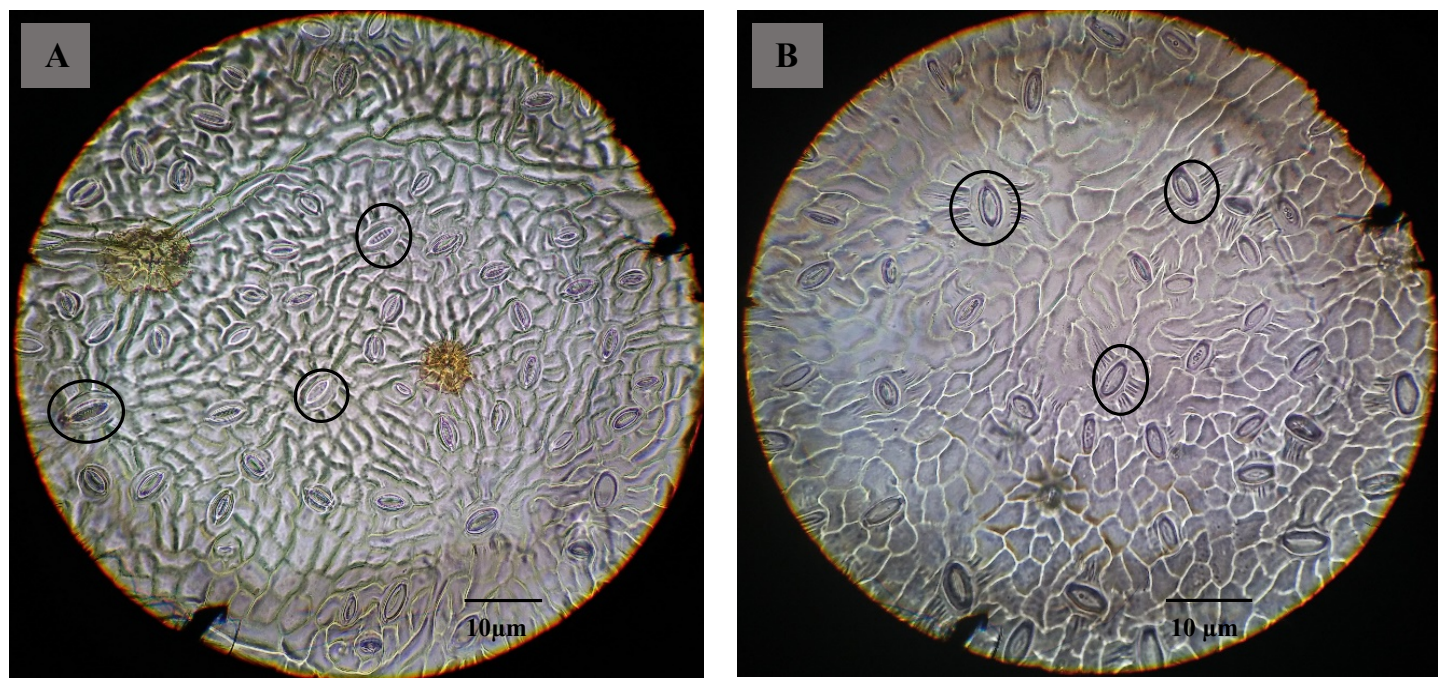

Fig. 3. Epidermis abaxial en foliolos de (A) Chandler y estomas.

Fig. 3. Abaxial epidermis in leaflets of (A) Chandler and (B) Franquette. Black circles indicate stomata.

Tabla 1. Severidad de la enfermedad en hoja y características estomáticas en los cultivares de nogal Chandler y Franquette.

Table 1. Leaf disease severity and stomatal characteristics in the walnut cultivars Chandler and Franquette.

\begin{tabular}{lcccc}
\hline Cultivar & SevH & DE & \multicolumn{2}{c}{ Estomas } \\
\cline { 4 - 5 } & $\mathbf{( \% )}$ & $\left(\mathbf{m m}^{-2}\right)$ & Ancho $(\mu \mathrm{m})$ & Longitud $(\boldsymbol{\mu m})$ \\
\hline Chandler & $19,29 \mathrm{a}$ & $387 \mathrm{a}$ & $16,46 \mathrm{a}$ & $28,86 \mathrm{a}$ \\
Franquette & $11,71 \mathrm{~b}$ & $315 \mathrm{~b}$ & $15,70 \mathrm{a}$ & $27,11 \mathrm{~b}$ \\
\hline
\end{tabular}

Letras distintas indican diferencias significativas según el Test de Student $(p<0,05)$.

Tabla 2. Severidad de la enfermedad en fruto y características de las lenticelas en los cultivares de nogal Chandler y Franquette.

Table 2. Fruit disease severity and characteristics of lenticels in the walnut cultivars Chandler and Franquette.

\begin{tabular}{|c|c|c|c|c|}
\hline \multirow[t]{2}{*}{ Cultivar } & \multirow{2}{*}{$\begin{array}{c}\text { SevF } \\
(\%)\end{array}$} & \multirow{2}{*}{$\begin{array}{c}\mathrm{DL} \\
\left(\mathrm{cm}^{-2}\right)\end{array}$} & \multicolumn{2}{|c|}{ Lenticelas } \\
\hline & & & Ancho $(\mu \mathrm{m})$ & Longitud $(\mu \mathrm{m})$ \\
\hline Chandler & 12,33 a & 131 a & 39,79 a & 58,57 a \\
\hline Franquette & $6,17 \mathrm{~b}$ & $113 \mathrm{~b}$ & 31,78 a & 43,89 a \\
\hline
\end{tabular}

Letras distintas indican diferencias significativas según el Test de Student $(p<0,05)$.

de ingreso del patógeno, luego, internamente aparecen otros factores que influyen sobre la tasa de infección, como los compuestos fenólicos que contiene el involucro del fruto, y pueden cumplir un rol importante en la susceptibilidad de la bacteria en diferentes cultivares (Solar et al., 2012; Shijiao et al., 2019).
Las yemas vegetativas y fructíferas juegan un rol central en la epidemiologia de Xaj, debido a que invade y se localiza en las escamas de las mismas a fines de la temporada anterior, donde sobrevive el reposo invernal, y en la primavera puede infectar las hojas, los amentos y las flores pistiladas (Lindow et al., 2014). Los cultivares 

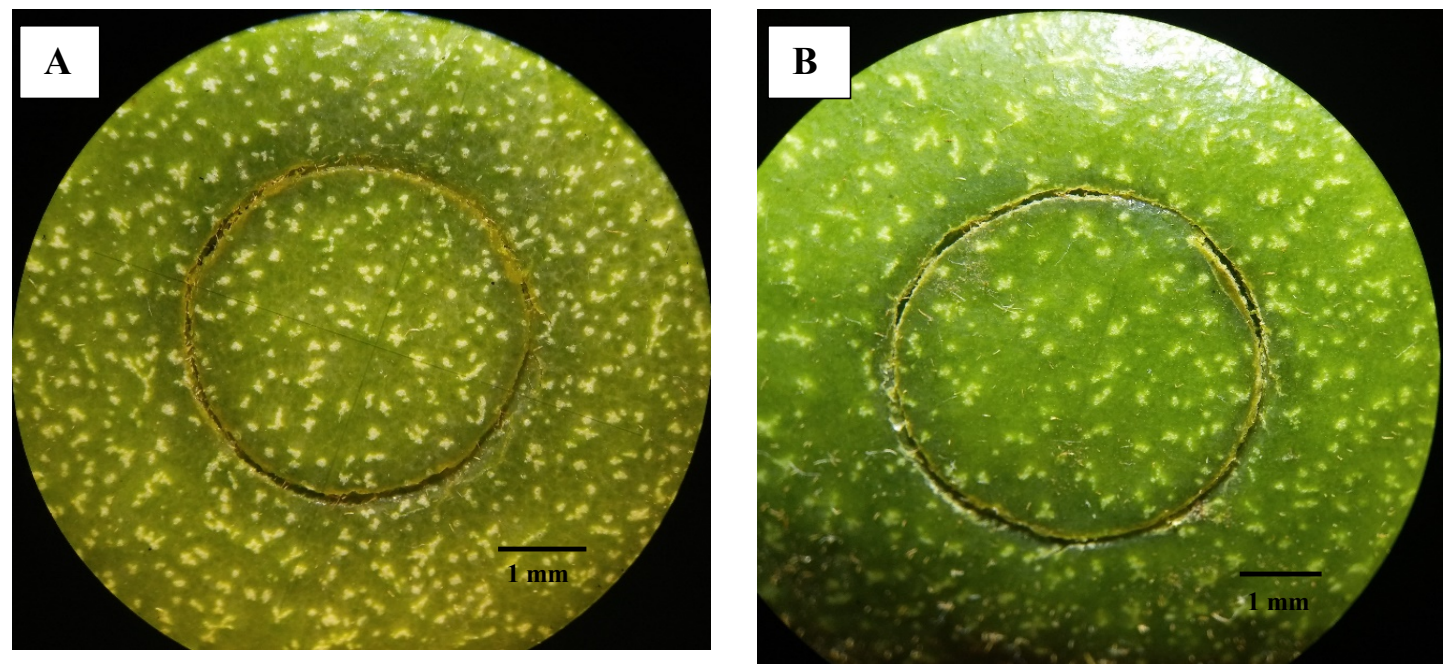

Fig. 4. Aspecto de las lenticelas desarrolladas sobre el fruto de (A) Chandler, y (B) Franquette. El círculo reperesenta el área de recuento y medición de las lenticelas.

Fig. 4. Appearance of the lenticels developed on the fruit of (A) Chandler and (B) Franquette. El circle represents the counting and measuring area of the lenticels.
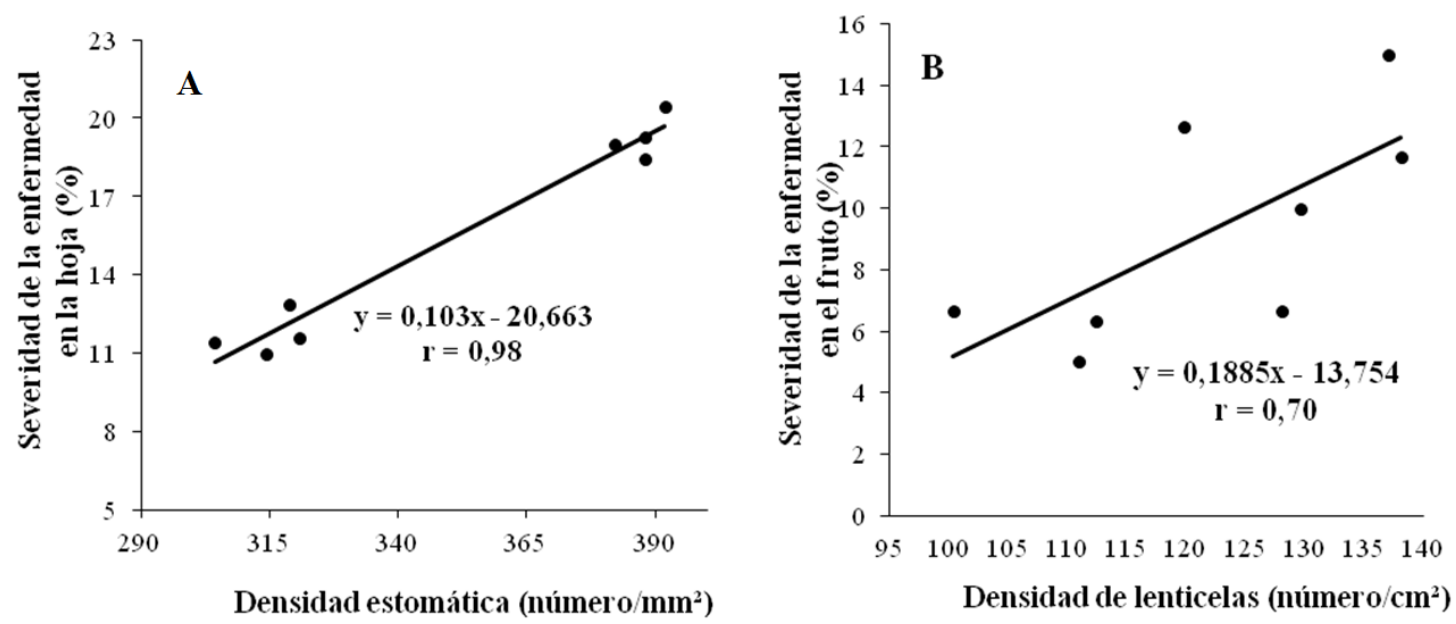

Fig. 5. A. Relación entre la severidad de la enfermedad en hoja y la densidad estomática de la cara abaxial en los cultivares de nogal Franquette (tolerante) y Chandler (suceptible). B. Relación entre la severidad de la enfermedad en fruto y la densidad de lenticelas de los cultivares de nogal Franquette (tolerante) y Chandler (susceptible).

Fig. 5. A. Relationship between leaf disease severity and stomatal density of the abaxial face in the walnut cultivars Franquette (tolerant) and Chandler (susceptible). B. Relationship between fruit disease severity and lenticel density of the walnut cultivars Franquette (tolerant) and Chandler (susceptible).

evaluados contrastan en el tipo de brotación, el cv. Chandler de brotación más temprana y el cv. Franquette de brotación tardía (Del Barrio y Martin, 2011). Esta diferencia en las fechas de brotación permitiría una mayor reproducción de la bacteria en el cv. de brotación temprana y generaría una mayor cantidad de inoculo secundario para las posteriores infecciones. Futuras investigaciones se centrarán en la cuantificación de las poblaciones de Xaj en los diferentes tejidos, en diferentes estadios fenológicos y su relación con las características anatómicas. 


\section{CONCLUSIONES}

Esta investigación analizó las características anatómicas de las hojas y los frutos de cultivares de nogal y relacionó estos rasgos con la severidad de la enfermedad peste negra, causada por Xanthomonas arboricola pv. juglandis. El menor número de estomas y lenticelas registrados en el $\mathrm{cv}$. Franquette correspondió con un menor daño observado en las hojas y en los frutos. Existe una relación entre la densidad de estomas en hojas y la densidad de lenticelas en fruto con la severidad de la enfermedad en los cultivares de nogal evaluados.

Los principales puntos de ingreso de la bacteria, estomas en las hojas y lenticelas en los frutos presentan contraste anatómicos entre cultivares clasificados como susceptible (Chandler) y tolerante (Franquette), por lo cual estos rasgos permitirían constituir un indicador de la susceptibilidad frente a la enfermedad más importante del cultivo de nogal.

\section{LITERATURA CITADA}

Adaskaveg, J.E., H. Förster, K. Nguyen, D. Thompson, D. Cary, L. Wade, et al., 2016. Epidemiology and management of walnut blight. Walnut Research Reports. California Walnut Board. http://fruitsandnuts.ucdavis. edu/files/259737.pdf (Accessed 30 May 2020).

Aleta, N., A. Ninot, C. Moragrega, I. Llorente, and E. Montesinos. 2001. Blight sensitivity of J. regia Spanish selections. IV International Walnut Symposium, Bordeaux, Francia. ISHS Acta Horticulturae 544:353-362. DOI: 10.17660/ActaHortic.2001.544.47.

Agrios, G. 2005. Fitopatología. Enfermedades de las plantas causadas por bacterias. Vol. 1. p. 532-542. 2 ${ }^{\mathrm{a}}$ ed. Limusa, México.

Belisario, A. 1996. Le principali malattie del noce in Italia. Informatore Fitopatologico. http://www.verdeepaesaggio.it/2008/02/04/ noce-principali-malattie-in-italia/ (Consulta $15 / 03 / 2020)$

Bouhier, R. 2017. El nogal de la Norpatagonia. $1^{\underline{a}}$ ed. p. 85-92. ISBN 978-887-521-8796. Ediciones INTA, Viedma, Rio Negro, Argentina.

Del Barrio R., y D. Martín. 2011. Aptitud agroclimática del Valle Inferior del Río Negro para los cultivos de avellano y nogal. Revista Pilquén, Sección Agronomía. 13(11). ISSN 1666-0587
Di Rienzo, J.A., F. Casanoves, M.G. Balzarini, L. González, M. Tablada, y C.W. Robledo. 2008. InfoStat, versión 2018. Grupo InfoStat, FCA, Universidad Nacional de Córdoba, Argentina.

Eskandari, S., R. Khakvar, and M. Torchi. 2018. Detection of bacterial blight of walnut (Xanthomonas arboricola pv. juglandis) in northwest of Iran and investigation of its pathogenicity on different native walnut cultivars. Biochem. Cell. Arch. 18(2):17851788. ISSN 0972-5075.

Fernandes, C., P. Albuquerque, R. Sousa, L. Cruz, and F. Tavares. 2017. Multiple DNA markers for identification of Xanthomonas arboricola pv. juglandis isolates and its direct detection in plant samples. Plant Dis. 101:858-865.

Flores, P., S. Seta, M. Gonzalez, R. Coniglio, S. Sferco, y A. Trevizán. 2004. Manejo químico y varietal de nogales frente a bacteriosis del nogal. Revista de Investigaciones de la Facultad de Ciencias Agrarias 4(5):25-31. Ed. Universidad Nacional de Rosario, Santa Fe, Argentina.

Frutos, D., G., López, M. López, C. Moragrega, A. Guevara, and A. Arieta 2008. Spreading model of Xanthomonas arboricola pv. juglandis on Juglans regia L. host selections grown in a shading shelter. Cost 873, WG and Management Committee Meeting, Athens, Greece.

Gallo, S. 2018. Control de bacteriosis del nogal (Xanthomonas campestris pv. junglandis). Ediciones Instituto Nacional de Tecnología Agropecuaria 11(76):1-3. Viedma, Río Negro, Argentina.

Giovanardi, D., S. Bonneau, and S. Gironde. 2016. Morphological and genotypic features of Xanthomonas arborícola pv. juglandis populations from walnut groves in Romagna region, Italy. Eur. J. Plant Pathol. 145:1-16. https://doi.org/10.1007/s10658-015-0809-2.

Hernández, L.F., and P.B. Green. 1993. Transductions for the expression of structural pattern: Analysis in sunflower. The Plant Cell 5:1725-1738.

Lamichhane, J.R. 2014. Xanthomonas arboricola diseases of stone fruit, almond and walnut trees: Progress toward understanding and management. Plant Disease 98(12):1602-1604. http://dx.doi.org/10.1094/ PDIS-08-14-0831FE.

Lang, M.D., and K.J. Evans. 2010. Epidemiology and status of walnut blight in Australia. J. Plant Pathol. 92 (1):S149-S155. 
Lindow, S., W. Olson, and R. Buchner. 2014. Colonization of dormant walnut buds by Xanthomonas arborícola pv. juglandis Is predictive of subsequent disease. Phytopathology 104(11):1163-1174. http:// dx.doi.org/10.1094/PHYTO-01-14-0001-R.

López, G., C. Frutos, and D. Frutos. 2011. Xanthomonas arborícola pv. juglandis damages in walnut (Juglans regia L.) leaves related to stomas density. Cost 873, WG3/WG4 Joint Meeting, Murcia, Spain. http://www.cost873. ch/5_activites/meeting__detail.php?ID=39 (Accessed 15 April 2020).

Melotto, M., W. Underwood, and S. Y. He. 2009. Role of stomata in plant innate immunity and foliar bacterial diseases. Annual Review of Phytopathology 46:101-122.

Morales, F. 2012. Composición fenólica, lipídica, actividad antioxidante y biodisponibilidad in vitro de 10 genotipos de nueces cultivados en la Región de Murcia. 280 p. Tesis Doctoral. Universidad Católica San Antonio, Murcia, España.

Muradoğlu, F., and M. Gündoğdu. 2011. Stomata size and frequency in some walnut (Juglans regia) cultivars. International Journal of Agriculture \& Biology ISSN Print: 15608530; ISSN Online: 1814-9596 11-238/ AWB/2011/13-6-1011-1015.

Naizaque, J., G. García, G. Fische, y L.M. Melgarejo. 2014. Relación entre la densidad estomática, la transpiración y las condiciones ambientales en feijoa (Acca sellowiana [O. Berg] Burret). Revista UDCA Actividad \& Divulgación Científica 17:115-121.

Nievas, W., M. Rossini, y J. Toranzo. 2014. Bacteriosis del nogal (Xanthomonas campestris pv. juglandis) en el Valle Medio del río Negro. Información Técnica. Ediciones INTA, Allen, Río Negro, Argentina, ISBN 978-987-521-481-1.
Temperini, C.V., A.G. Pardo, y G.N. Pose 2014. Bacteriosis y necrosis apical del nogal: Complejos microbianos involucrados en la caída temprana de frutos en el Valle Medio del Río Negro. Revista SENASA (5-6):9-14.

Temperini, C.V., A.G. Pardo, and G.N. Pose. 2017. First report of apical necrosis in walnut cultivars from Northern Argentina Patagonia. J. Plant Pathol. Microbiol. 8:414. doi: 10.4172/2157-7471.1000414.

Shijiao, J., H. Shan, H. Dan, C. Guangli, F. Ke, X. Xiao, et al. 2019. The accumulation of phenolic compounds and increased activities of related enzymes contribute to early defense against walnut blight. Physiological and Molecular Plant Pathology https://doi. org/10.1016/j.pmpp.2019.101433. (Accessed 10/05/2020).

Spielman, L.J., M. Hubbes, and D. Lin. 1986. Septoria musiva on hybrid poplar in Southern Ontario. Plant Disease 70:968-971.

Solar, A., J. Jakopoc, R. Veberic, and F. Stampar. 2012. Correlations between Xanthomonas arborícola pv. juglandis severity and endogenous juglone and phenolic acids in walnut. Journal of Plant Pathology 94(1):229235.

Sorokopudov, V.N., T.C. Nguyen, S.N. Shlapakova, and T.B. To. 2015. Some features of the species of the genus Juglans $\mathrm{L}$ in the conditions of bryansk oblast. ISJ Theoretical \& Applied Science 23(3):49-54. http://dx.doi. org/10.15863/TAS.2015.03.23.10. 